\title{
Location accuracy limitations for CCD cameras
}

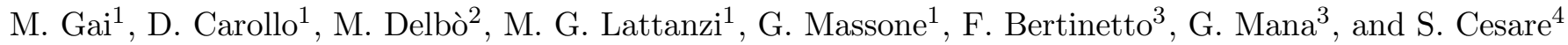 \\ 1 Osservatorio Astronomico di Torino, Str. Osservatorio 20, 10025 Pino T.se (TO), Italy \\ e-mail: gai@to.astro.it; carollo@to.astro.it; lattanzi@to.astro.it; massone@to.astro.it \\ 2 Deutsches Zentrum für Luft- und Raumfahrt, Berlin, Germany \\ e-mail: marco.delbo@dlr.de \\ 3 Istituto Metrologico "G. Colonnetti" del CNR, Str. delle Cacce 73, 10135 Torino, Italy \\ e-mail: f.bertinetto@imgc.to.cnr.it; g.mana@imgc.to.cnr.it \\ 4 Alenia Spazio, C.so Marche 41, 10146 Torino, Italy \\ e-mail: scesare@to.alespazio.it
}

Received 27 September 2000 / Accepted 28 November 2000

\begin{abstract}
The accurate measurement of the position of celestial objects is a fundamental step for several astrophysical investigations. For ground based instruments, the atmosphere is considered the basic limiting factor; in space, the knowledge of the instrumental parameters and/or of their stability define the performance limits, but CCD cameras operated in time delay integration may take advantage of their operating mode to reduce significantly the calibration problem. We implemented a low-cost laboratory experiment aimed at assessing the precision achievable in the location determination with a CCD camera, by evaluating the measurement repeatability throughout a set of images of a simulated stellar field. Our experiment provides an initial location dispersion of the order of $1 / 100$ of the CCD pixel, with clear evidence of dominant common mode effects. After removing such terms with straightforward numerical procedures, we achieve a final location precision of 1/700 pixel on individual images, or 1/1300 pixel on co-added images. The scaling of precision with target magnitude is in quite good agreement with theoretical expectations. The initial common mode systematics appear to be induced by the thermal control of the CCD camera head, which degrades the structural stability. In actual implementations, such problems can be greatly reduced by proper design. Finally, our results show that residual effects, which could hamper the final astrometric accuracy, can be calibrated out with simple procedures.
\end{abstract}

Key words. instrumentation: detectors - methods: data analysis - space vehicles - techniques: image processing - astrometry

\section{Introduction}

Astrometric measurements of celestial objects are often based on images obtained with CCDs. These measurements allow accurate determination of relative positions as well as, through the direct determination of parallaxes, of the tri-dimensional perspective of regions within our Galaxy, which are becoming increasingly large as measurement precision increases. With the success of the ESA astrometric mission Hipparcos (Perryman 1997), space astrometry has come of age, establishing itself as the most appropriate means for exploiting future micro-arcsec precision measurement capabilities.

Both ESA and NASA are funding three ambitious initiatives in global space astrometry. The measurement principle of the Space Interferometry Mission (SIM, Shao 1998), is somewhat different from that used on Hipparcos, requiring the combination of afocal beams from a single

Send offprint requests to: M. Gai source at one time (Michelson interferometry) rather than the simultaneous imaging of a star field. The other two missions, FAME (Horner et al. 1998) and GAIA (Gilmore 1998), are direct evolutions of the Hipparcos measurement concept; basic location measurements are accomplished on focal planes with large angular size, using mosaics of CCDs. As we are more familiar with the GAIA mission, we will reference to that for some of the practical implications of our findings; details on the focal plane architecture of the GAIA detector have been presented recently (Saint Pé 1999). However, we believe that many of the results presented in this article should also be of interest to the FAME community.

The GAIA measurement concept is, as for Hipparcos, the complete and repeated coverage of the sky by a scanning satellite, providing accurate astrometry by reduction of the endless strip obtained by its CCD detectors operating in Time Delay Integration (TDI) mode. Given ideal optics, attitude and detectors, the location accuracy of 
the targets within the image, and therefore of the angular position on the sky, is limited only by photon statistics. In particular, for a given imaging system, in stable conditions, the expected location dispersion $\sigma$ for a point-like target image is (Lindegren 1978):

$$
\sigma \geq \frac{\lambda}{4 \pi L S N R} \quad \rightarrow \quad \sigma=\alpha \frac{\lambda}{4 \pi L S N R}
$$

where $L$ is the characteristic width of the aperture (i.e. the variance of the telescope aperture function in the ordinary statistical sense), $S N R$ is the signal to noise ratio of the target, and $\lambda$ is the monochromatic wavelength used for observation (or a suitable effective wavelength for a finite passband). The factor $\alpha \geq 1$ takes into account the system geometry (i.e. optics aberrations), the pixel matching of stellar images (sampling), the applied location procedure, and different sources of noise, each term introducing a performance penalty in the location estimate (Gai et al. 1998; Lattanzi et al. 1997).

At the bright end of the measured objects (actual values depend on the saturation level of the detection system adopted), the intrinsic data dispersion appears to be a very small fraction of the detector pixel size, or of the image size $(\sim \lambda / L)$, as a natural consequence of the very high $S N R$. In order to ensure photon-limited performances to GAIA, neglecting other error sources (as, e.g., attitude disturbances), the elementary image location process should provide an intrinsic dispersion of better than $1 / 1000$ of the CCD pixel for the brightest targets $(V \sim 10-11 \mathrm{mag})$. It is reasonable to raise the suspicion that, at this level, the discrepancy between the physical behavior of the device and the simple geometric model assumed in the above analysis becomes significant.

Indeed, ground based observations quote, in favorable conditions, a location accuracy on individual images of the order of 1/100 pixels (Smart et al. 1999), although such precision is believed to be limited mainly by atmospheric effects. It is therefore important to address the problem of the kind of accuracy attainable in a real system which is not dominated by atmospheric turbulence, thus approximating operations in space. Real-world CCD characteristics (like deviation from uniformity or from linear pixel response) are in many cases sufficiently well known to allow a more detailed device modeling than required herein. This would provide further insight into the ultimate performance achievable, and, more importantly, would help define operation and calibration requirements for optimal astrometric results. Such a higher level of CCD modeling, and above all the calibration issues, will be the subject of further investigations.

Hereafter, Sect. 2 describes the equipment we used to generate sets of frames, on which the statistics of Eq. (1) can be directly evaluated; Sect. 3 describes the operating concepts of GAIA leading to our design; in Sect. 4, the data treatment is described; Sect. 5 investigates on the systematic effects evidenced in our simple set-up; in Sect. 6, the implications of our findings for the operation

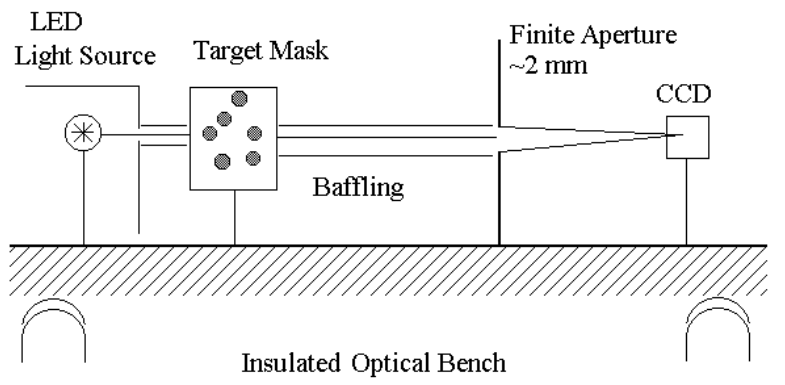

Fig. 1. Schematic of the experimental set-up: from the left, the source system generating the simulated stellar field, imaged by the doublet on the CCD camera

of the GAIA focal plane are described; finally, in Sect. 7, we draw our conclusions.

\section{Experimental set-up}

The key concept under investigation is the limiting location accuracy of a CCD camera when acquiring the image of a set of point-like sources. Therefore, we minimize by design the sensitivity to system perturbations, i.e. optical aberrations, mechanical and thermal disturbances. Figure 1 illustrates the experiment in its essential parts, mounted on an optical bench to ensure some degree of stability to the optical system, whereas Fig. 2 shows the set-up as installed in the laboratory. The simulated stellar field produced by the equipment is shown in Fig. 3. Seven significant sources are imaged on the detector. Reference to individual sources is done below according to the numbers in Fig. 3. The experiment was mounted in the Alenia Spazio laboratories (Torino). The data sets

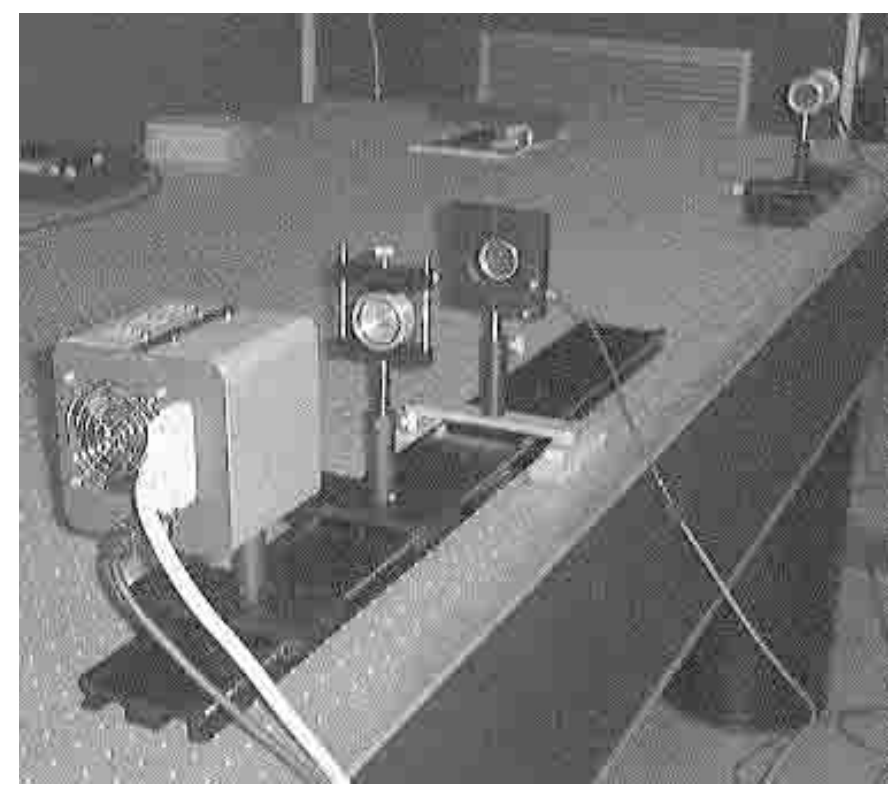

Fig. 2. The experimental set-up: bottom-left to top-right, the CCD camera head, the doublet optics and aperture stop, the shutter (decoupled from the bench), the source system with target mask, frosted glass diffuser and LEDs. Baffling has been removed 
Table 1. The letter $I$ indicates the intensity of the seven stars relative to star No. 4 (faintest), Mag. is the magnitude relative to No. 2 (brightest); $X$ and $Y$ are the frame coordinates of the locations of the star-like images, as derived by averaging over the whole set of measurements; $\sigma_{X}$ and $\sigma_{Y}$ are the image widths (standard deviations of the photon distribution) calculated from sequence No. 1

\begin{tabular}{ccccccc}
\hline Star & $I$ & Mag. & $\begin{array}{c}X \\
\text { [pixel] }\end{array}$ & $\begin{array}{c}Y \\
\text { [pixel] }\end{array}$ & $\begin{array}{c}\sigma_{X} \\
\text { [pixel] }\end{array}$ & $\begin{array}{c}\sigma_{Y} \\
{[\text { pixel] }}\end{array}$ \\
\hline 1 & 14.58 & 0.02 & 197.321 & 283.297 & 1.453 & 1.398 \\
2 & 14.82 & 0.00 & 249.322 & 191.296 & 1.460 & 1.418 \\
3 & 13.63 & 0.09 & 315.330 & 292.275 & 1.432 & 1.369 \\
4 & 1.00 & 2.93 & 317.571 & 428.218 & 1.396 & 1.357 \\
5 & 3.76 & 1.49 & 103.547 & 305.166 & 1.430 & 1.381 \\
6 & 3.23 & 1.65 & 218.529 & 101.113 & 1.469 & 1.426 \\
7 & 3.53 & 1.56 & 434.523 & 222.105 & 1.472 & 1.419 \\
\hline
\end{tabular}

analyzed herein were collected on December 2nd, 1998, and some preliminary results have been recently presented (Gai et al. 1999).

The light source is a light emitting diode (LED) circuit. A frosted glass flat generates a uniformly scattered light beam, illuminating the artificial stellar field, a mask of pinholes, each simulating a point-like source at infinity. The pinhole separation $s$ is large compared to diameter $d$, to provide a field with limited star density and well separated images. The distance to the CCD camera is $r=1 \mathrm{~m}$, the pinhole diameter is $d=30 \div 100 \mu \mathrm{m}$, and the typical pinhole separation is $s \sim 10 \mathrm{~mm}$. Therefore, $d / s \sim 10^{-2} \ll 1$; the intrinsic angular size of the sources at the camera is $\theta_{\mathrm{d}}=d / r=6^{\prime \prime} \div 20^{\prime \prime}$, and their angular separation is $\theta_{\mathrm{s}}=s / r \simeq 30^{\prime}$.

We find that sources $\{1,2,3\}$ are brightest, with comparable magnitude, No. 4 is the faintest, whereas sources $\{5,6,7\}$ have intermediate intensity. Table 1 provides the intensity and magnitude values, the $X$ and $Y$ source position (in pixels, averaged over the frame set) and the image width (in pixels, for sequence No. 1). The brightest source, No. 2, has been set to magnitude 0 , whereas the relative intensity is referred to the star No. 4, for convenience; the measured magnitude spread is $\Delta m=2.9 \mathrm{mag}$.

The aperture stop diameter of the camera system is $D=2 \mathrm{~mm}$, resulting in an Airy disk diameter $\Theta_{\mathrm{A}}=2^{\prime} .5$; the simulated stars are therefore completely unresolved, as $\theta_{\mathrm{d}} / \Theta_{\mathrm{A}} \ll 1$. Due to the small aperture, the matching optics (a doublet) is used in a small region close to its optical axis, reducing the sensitivity to aberrations and mechanical tolerances. The internal shutter of the CCD camera is replaced by an external device, decoupled from the optical bench, to suppress a potential source of vibrations within the equipment.

The CCD camera used in our experiment is model HR 1600 from DTA (Italy), using a Kodak KAF-1600 chip, with $9 \times 9 \mu \mathrm{m}$ pixels, cooled by a Peltier cell to an operating temperature of $-5{ }^{\circ} \mathrm{C}$. The detector is a thick,

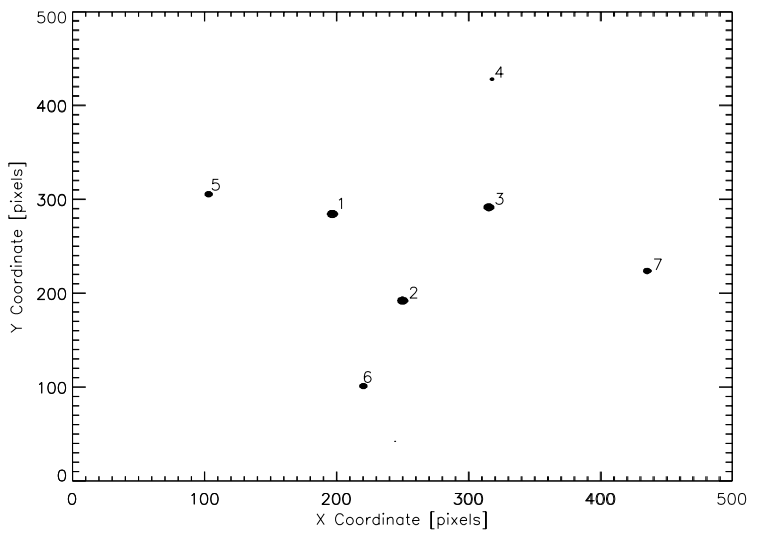

Fig. 3. The simulated star field; sources numbered counterclockwise from the center. In the text, these labels are used as reference for the individual sources

front illuminated CCD with format $1536 \times 1024$ pixels; its quantum efficiency is $Q E=35-40 \%$ in the spectral region of interest. The read-out electronics is based on a 16 bits analog to digital converter (pixel period $20 \mu \mathrm{s}$ ), with correlated double sampling. This camera was adopted because readily available, and because of its geometric similarity with the selected detectors for the baseline option of GAIA (Saint Pé 1999).

\section{Some advantages of the TDI mode}

In TDI, the motion of the image on the focal plane must be matched by the CCD clock rate: each potential well follows the current position of the associated point in object space, observed by the optical system. The continuous motion is matched to a step-by-step process, since the CCD potential well is displaced by one pixel per clock cycle. Ideally, the conventional CCD pixel, associated to a specific device location, is replaced by a logical pixel generated by the superposition of the contributions from all subsequent steps of elementary exposure. Each logical pixel scans all physical electrodes along one CCD column, averaging all local variations over the whole device. The sensitivity of both photometric and astrometric performance to local device parameters is therefore reduced. The geometric calibration of a CCD used for pointed observation, in principle, requires characterization of every pixel, which is an heavy task for the large logical format $N_{1} \times N_{2}$ of most modern devices. For a CCD in TDI mode, thanks to the uniformity of logical pixels from each CCD column, the number of individual parameters drops from the order $N^{2}$ to $\sim N$, i.e. the linear size of the device.

A relevant case of effects due to local CCD characteristics has recently been investigated in detail (Anderson \& King 1999), concerning the astrometric and photometric calibration of the Wide Field/Planetary Camera 2 (WFPC2) on board the Hubble Space Telescope (HST). The detector is affected by a manufacturing defect, so that one row out of every 34 is $3 \%$ narrower than the design value. The smaller pixels collect less light, and the 
reconstructed image is compressed; the irregular effect is due to the pointed observations. For drift scanning observations, the resulting image would be composed of equal logical pixels with equivalent width $\sim 1-3 \% / 34=0.999912$ of the nominal value, effectively suppressing the lack of uniformity to a very high degree. The localized astrometric error of the pointed case is spread out uniformly in TDI observation, allowing accurate calibration thanks to the measurement technique: for GAIA, images of the same targets in subsequent revolutions must superpose each other, providing the image scale and therefore the correction to the actual value of the logical pixel size. Given the large number of bright stars on each scan circle, very accurate calibration of the pixel scale is achievable: using $10^{3}$ bright stars, with average precision $1 / 300$ pixels, the precision is $\sim 310^{-3} / \sqrt{10^{3}} \simeq 10^{-4}$.

We based our experiment on the acquisition of static images, as this represents a worst case, able to provide a conservative evaluation of the potential performance of a CCD in TDI mode. Pointed exposures may be limited by irregularity of the pixel geometry (e.g. due to the CCD manufacturing process) or by pixel to pixel response variation, requiring very good detector calibration from a geometric standpoint. Assuming uniform pixel size and response, local variation effects are cumulated in the residual errors, which provide an upper limit to the potential performance achievable either in TDI operation (which benefits of pixel equalization) or by a pointed instrument with very good detector characterization. In this sense, a simple set-up is sufficient to yield the desired information, at least concerning the geometric aspects of the CCD and the trend with SNR; implementation of a TDI test is much more complex and expensive, and it is affected by potential limitations which are beyond the scope of our current investigation. For example, requirements for the timing of focal plane electronics have been evaluated by the authors (Gai et al. 1997); the additional performance penalty due to real device limitations (e.g. charge transfer efficiency, CTE, and its progressive degradation for radiation damage in the space environment) are under study with special reference to GAIA (Lindegren, private communication). The trap/hot pixel map should be updated frequently; hot pixels can be reduced or removed by warming up the CCD (Holtzman et al. 1995), whereas the effect of traps can be reduced by the "fat zero" techniques if low signal sensitivity is not critical. For the HST WFPC2, CTE induces signal losses which require correction dependent on the $X$ and $Y$ position of the target in the frame (Whitmore et al. 1999). TDI observation remove the effect on the along scan direction, as all pixels are transferred over the whole array.

In principle, TDI only equalizes the CCD response in the along scan direction, which is the fundamental measurement direction in the Hipparcos-like concept used by both GAIA and FAME. The requirements on across scan direction are much less stringent, because we need to separate different nearby sources and possibly to provide a first-order position for data reduction and attitude reconstruction. Calibration is eased by the redundant mosaic structure of the focal plane: every target crosses several CCDs, providing independent values of position associated to the satellite motion and to the detector geometry, which become measurable. Detailed modeling of the detector, its operation and of the available options for data quality assessment are crucial aspects of calibration and data reduction.

\section{Data reduction and analysis}

For seven different signal levels, sets of 50 frames are collected, providing a statistically significant sample of images in the same nominal conditions.

Each image is approximated by a bidimensional Gaussian profile; a least-squares fit provides estimates of background, intensity, characteristic width $\sigma$, and center coordinates. Given the mismatch in the fitting function with respect to the Point Spread Function (PSF) of the unobstructed circular aperture, and the sampling resolution of $\sim 7$ pixels per Airy disk, the location performance is degraded by approximately $11 \%$ (Gai et al. 1998). Since several parameters are estimated from the image, the location process is not optimal, so that the positional accuracy is further degraded, as discussed below. For GAIA, independent measurements of star brightness and position are taken in each transit. The diameter of the PSF Airy disk is $\sim 5$ times the variance $\sigma$ of the image profile; the latter is used hereafter as the characteristic width of the image. Its average value for the frame sequence No. 1 is listed in Table 1. We do not apply corrections for dark current, bias, and flat field: this gives a worst case result. Figure 4 shows, for a set of 50 frames obtained with 18 s exposures, a typical behavior of the system: the positions of the three central sources (labeled in Fig. 3 as 1, 2 and 3) are plotted by the solid, dotted and dashed line, respectively; the $X$ and $Y$ coordinates are shown in the top and bottom plot, respectively. The three source positions are shown after subtraction of their average value within the frame set, in order to display them on the same plot; a significant correlation among the target coordinates is evident on both axis, as all the targets are affected by a common mode disturbance and feature a much smaller dispersion with respect to the common trend. Also, the $X$ and $Y$ coordinates do not appear to have a linear correlation, but they feature similar time scale and amplitude. The same considerations apply to the other four stars (4, 5, 6 and 7 in Fig. 3), not shown here for clarity. We restrict temporarily our analysis to the central three stars.

For each frame $(n=1, \ldots, 50)$, we compute the Gaussian center coordinates $\left(x_{\mathrm{G}}(T, n), y_{\mathrm{G}}(T, n)\right)$ of the three stars $(T=1,2,3)$, as described above; the standard deviation of the raw data is slightly above $1 \%$ of the pixel 

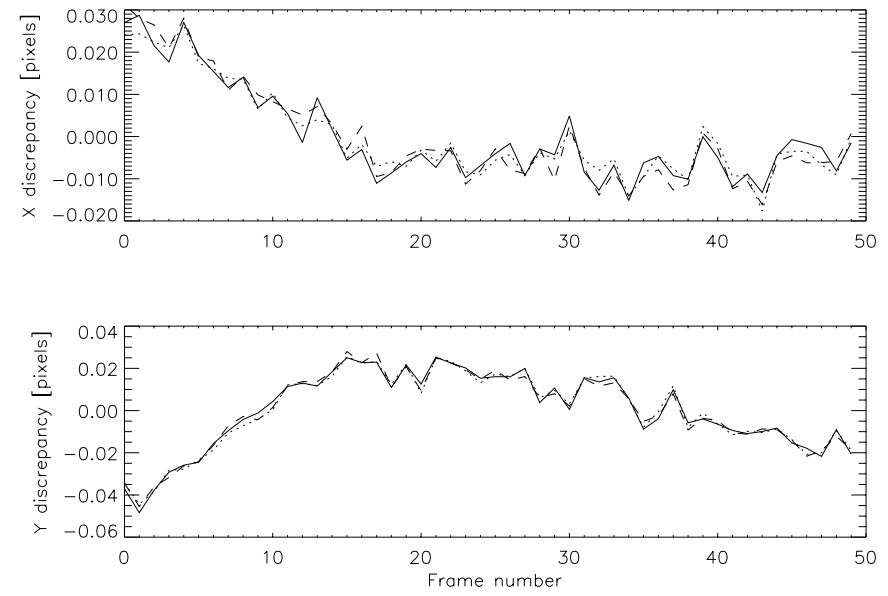

Fig. 4. Frame positions for the three central artificial stars versus frame number; data set No. 2, $18 \mathrm{~s}$ exposures. The individual positions are referred to the mean location computed by averaging over the whole set of frames, to allow superposition of the plots. Sources Nos. 1, 2, and 3 are represented by the solid, dotted and dashed lines, respectively; top: $X$ coordinate; bottom: $Y$ coordinate

size. We evaluate the average "center of mass" of the three stars over the 50 frames, with equal weights, as

$x_{\mathrm{cm}}(n)=\frac{1}{3} \sum_{T=1}^{3} x_{\mathrm{G}}(t, n) \quad y_{\mathrm{cm}}(n)=\frac{1}{3} \sum_{T=1}^{3} y_{\mathrm{G}}(t, n)$,

and define its average value throughout the measurement:

$\left\langle x_{\mathrm{cm}}\right\rangle=\frac{1}{50} \sum_{n=1}^{50} x_{\mathrm{cm}}(n) \quad\left\langle y_{\mathrm{cm}}\right\rangle=\frac{1}{50} \sum_{n=1}^{50} y_{\mathrm{cm}}(n)$.

The photo-center displacement is retained as estimate of the observed common mode motion of the images. Then, we define new (reduced) coordinates $\left(x_{\mathrm{R}}(T, n), y_{\mathrm{R}}(T, n)\right)$ by subtracting the estimated displacement:

$x_{\mathrm{R}}(T, n)=x_{\mathrm{G}}(T, n)-x_{\mathrm{cm}}(n)+\left\langle x_{\mathrm{cm}}\right\rangle$,

$y_{\mathrm{R}}(T, n)=y_{\mathrm{G}}(T, n)-y_{\mathrm{cm}}(n)+\left\langle y_{\mathrm{cm}}\right\rangle \cdot$

The new data set is shown in Fig. 5. The dispersion of the reduced coordinates reaches $1 / 850$ pixel, about 10 times less than the original data, and the correlation among targets is removed quite effectively.

The photo-center evaluation factors out a fraction of the intrinsic motion of each target. With comparable standard deviation of the parent data, as justified by the similar source intensity, i.e. $\sigma\left(x_{\mathrm{G}}(m, n)\right) \simeq \sigma_{\mathrm{G}}(x)$, where $m=1,2,3$ refers to the stars and $n=1,2, \ldots, 50$ refers to the frames, we get the dispersion of the reduced coordinates: $\sigma\left(x_{\mathrm{R}}(m, n)\right) \simeq \sqrt{2 / 3} \sigma(x)$. The same holds on $Y$ axis. The measured standard deviation of the reduced coordinates is associated to a standard deviation of the parent data $\sim 1 / 700$ pixel.
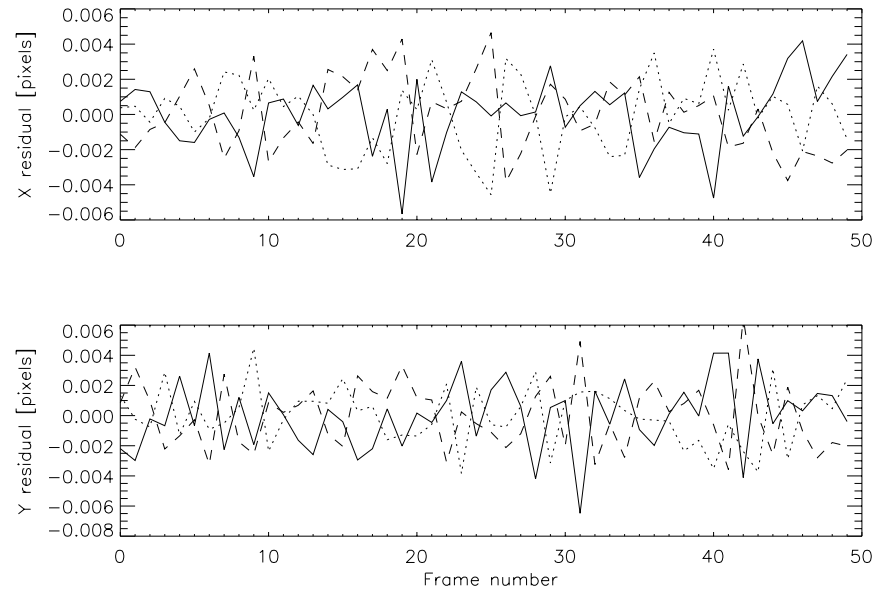

Fig. 5. Reduced coordinates of stars 1, 2, and 3 (represented by the solid, dotted and dashed lines, respectively) vs. frame number, for the same data set as in previous Fig. 4; top: $X$; bottom: $Y$. Common mode is strongly suppressed and the residual fluctuations are reduced by about one order of magnitude

For the case of $N$ sources of comparable brightness, the two sets of coordinates have quite similar precision, because the transformation factor is

$\frac{\sigma(x)}{\sigma\left(x_{\mathrm{R}}\right)} \simeq \sqrt{\frac{N}{N-1}} \simeq 1+\frac{1}{2 N}$.

Removal of the common image shift, estimated from the data, appears to be a convenient strategy for correction of common mode errors, e.g. due to jitter in the satellite attitude, at a reasonable statistical cost. The analysis can be applied to the whole set of seven targets, with appropriate weighting of the data by the expected variance, i.e. based on SNR. In the case of GAIA, thousands of targets are observed at any time; this appears to be a promising condition for effective monitoring of the instrument stability and data calibration.

\subsection{Scaling of accuracy vs. magnitude}

It is possible to reverse Eq. (1), using the measured position dispersion, image size and SNR to deduce the instrumental degradation factor:

$\alpha=4 \pi \frac{L}{\lambda} \sigma S N R$.

Considering the whole data set, we get the average value of the degradation factor $\alpha=2.206$, with standard deviation 0.392 , in the reduced coordinates $(\alpha=2.694$ in parent frame coordinates). This value is larger than expected only from sampling resolution and PSF model mismatch, however a large common mode error has been subtracted, reducing the data dispersion by about one order of magnitude, so that we can expect a significant residual unmodeled error at this level. No evidence appears of a significant trend depending on the signal level, i.e. target magnitude. It appears that, within the scope of our experiment, the centering precision is not limited by the 


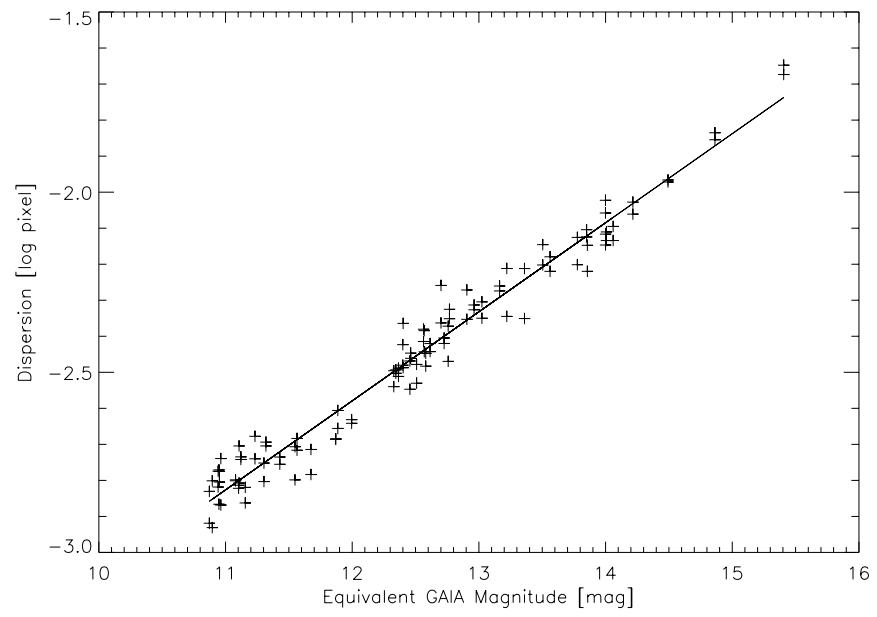

Fig. 6. Accuracy of reduced coordinates vs. instrumental magnitude (logarithmic plot of the location standard deviation). All targets and data sets are included, and the reduced coordinates are evaluated by subtraction of the photo-center motion, defined by the weighted average of the star positions in each set. The weight is the target intensity. The solid line represents the best linear fit of the experimental values: $\log \sigma=-5.55+0.247 m$

detector geometry, or at least that all unmodeled effects do not dominate with respect to the precision specified for GAIA, in terms of pixel fraction. The results can also be expressed in terms of residual data dispersion versus the instrumental magnitude (Fig. 6). For bright targets, the location error from Eq. (1) depends on the square root of the signal intensity, so that we can reformulate the relationship as $\log \sigma=k_{1}+k_{2} m$, where $k_{1}$ is an instrumental factor and $k_{2}=0.2$. The change from reduced to frame coordinates only affects the additive term, without influencing the scaling vs. magnitude. The linear fit of the experimental data (solid line in Fig. 6) provides an instrumental magnitude $k_{1}=-5.55 \pm 0.05$, and a scaling factor $k_{2}=0.247 \pm 0.004$ instead of 0.2 . The intrinsic dispersion of the latter parameter is smaller than its discrepancy with respect to the theoretical value, suggesting significant residual systematics in our error model.

The choice of the magnitude scale is in approximate agreement with the baseline GAIA design parameters: the elementary exposure of a target of magnitude $V=15$ and near-solar type provides a signal of $\sim 3.910^{4}$ photoelectrons at the focal plane, and our magnitude is scaled accordingly. Throughout the measurement range, the scaling of location accuracy with $S N R$ (or magnitude) is reasonably linear, in spite of comparably large variations of the environment and of some operating parameters.

To explore a larger parameter space, we implement two tests, frame binning and coadding. In the former case, we reduce the image resolution by summing the pixels inside each $2 \times 2$ box; the $500 \times 500$ frame is reduced to $250 \times 250$ larger pixels. Each star is now sampled over little more than $3 \times 3$ pixels, and the same location process is applied, with an expected dispersion corresponding to
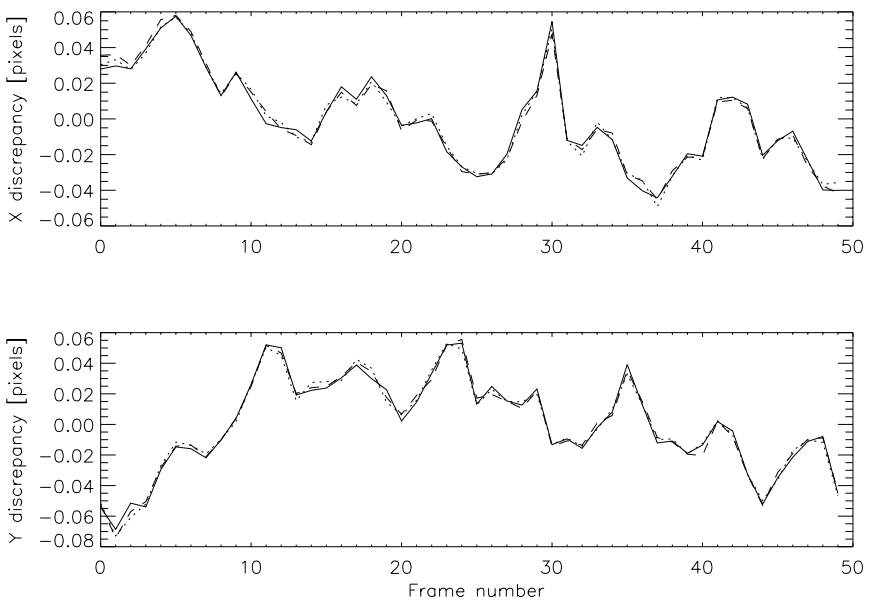

Fig. 7. Frame positions for the three central stars versus frame number; data set No. 5, $25 \mathrm{~s}$ exposures. Individual positions are referred to the average star location. Sources 1, 2, and 3 are represented by the solid, dotted and dashed lines, respectively; top: $X$ coordinate; bottom: $Y$ coordinate

a smaller fraction of the larger pixels. The degradation due to reduced resolution is about $35 \%$. The evaluation of the new data set provides at best a centering accuracy of $1 / 1030$ new pixels, consistent with the scaled geometry.

We also compress the data set by generating a new set of 25 frames, each obtained by the sum of two original frames, pixel by pixel, retaining the original resolution. This process is similar, in principle, to doubling the exposure time, with a net increase of the SNR by a factor $\sqrt{2}$. The location accuracy achieved by the new set of coadded frames reaches $1 / 1100(X)$ and 1/1300 $(Y)$ pixels. The instrumental factors are quite compatible with the original values: the instrumental magnitude becomes $k_{1}=-5.51 \pm 0.07$, and the scaling factor is $0.248 \pm 0.006$. The instrument degradation factor is now $\alpha=2.3 \pm 0.5$.

\subsection{Image stability and system perturbations}

The evolution of the experimental conditions is monitored by the system: in particular, the $X$ and $Y$ image widths are evaluated on each image. Within each image set, the variation of the image width is of the order of $1 / 100$ pixel or smaller, comparable with the estimate error. A significant variation of the $X$ image width after the third frame sequence seems to be due to a system transition, associated to the air conditioning system, off during the first three sequences, and switched on at that point, with an interruption of one hour to allow for temperature settling. No similar variation is observed for the $Y$ coordinate, and this may be due to the mounting geometry.

An insight on the variation origin is offered by the data: Fig. 7 shows the $X$ (top) and $Y$ (bottom) center coordinates of stars 1, 2 and 3 (solid, dotted and dashed line, respectively) throughout frame sequence no. five, taken after turning on the air conditioning system. Again, we subtract the average value of position to each source, to 
superpose the plot. The standard deviation of the target positions is $\sim 2 \%$ pixel, with large common mode perturbations. The residuals (after subtraction of the common mode motion, evaluated on the seven stars) are at the level of $1 / 600$ pixels. Oscillating fluctuations with a period of approximately 11 frames (about eight minutes) are evident, whereas without the air conditioning system (Fig. 4) we perceive a settling phase of approximately 18 frames ( $\sim 15 \mathrm{~min}$ ), before reaching a stationary regime, still affected by fluctuations and a slow drift. All sequences observed with air conditioning on are affected by similar oscillations, with comparable time constant; also, all frame sets taken with air conditioning off feature a slow drift. Therefore, the air conditioner interacts with the source of the perturbations.

The most likely origin of the perturbations is the CCD camera head itself, which is the only subsystem featuring significant increase in activity during operation. This hypothesis is analyzed in more detail in the next section, deriving a few numbers which can be compared with the requirements for GAIA.

\section{Origin of perturbation}

The time scale of the images is between $30 \mathrm{~s}$ and one minute per frame. High frequency disturbances, with period significantly shorter than the elementary exposure time, are averaged out by the analog integration; only noise acting on a time scale comparable with the single exposure, i.e. with characteristic frequency $<10^{-1} \mathrm{~Hz}$, may provide a significant residual.

The mechanical set-up has been designed for high stiffness, to achieve high stability; the proper frequencies of individual components are of order of hundreds of $\mathrm{Hz}$, and it is reasonable to expect for the lowest global proper frequency a value of a few tens of $\mathrm{Hz}$. The time scale of $\sim 10^{2} \mathrm{~s}$ is typical of the thermal evolution of a small size system, sensitive to any parameter affecting the conduction, convection and radiative transfer properties toward the environment. Usually, analysis can not be carried on explicitly except for very simple systems, and the designer is forced to work on a finite-element model (FEM) and evaluate the system behavior by means of numerical simulators. Some effort have been spent in our design to reduce the coupling within the set-up and with the external world, by means of some shielding, and by mounting the equipment over an optical bench with large thermal mass.

The generation of small oscillations is often associated to digital control systems, because of the finite resolution associated with the quantization levels. Hereafter, we describe a simplified model of an heating system, sufficient to illustrate the mechanism; for the CCD head, the sign of heat flow is reversed, because it is a cooling system. The system, shown in Fig. 8, is represented by the thermal mass $M$, to be stabilized at temperature $T_{\mathrm{r}}$; the environmental conditions are such that in the temperature interval around $T_{\mathrm{r}}$ the average power dissipation to the environment is $P_{\mathrm{E}}$. The thermometer measures the current

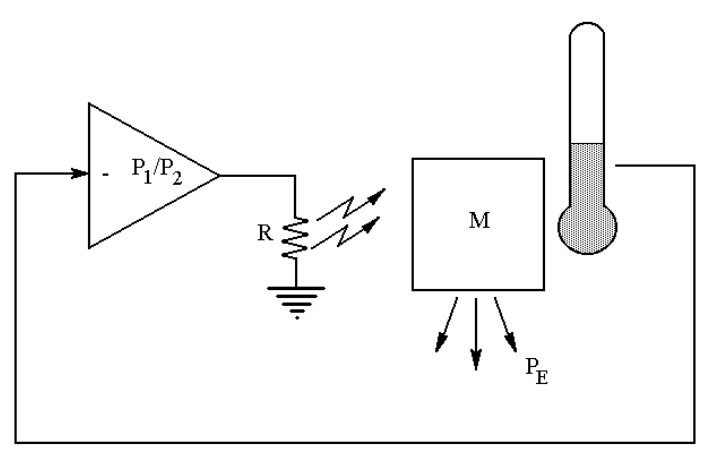

Fig. 8. A simple thermal control system: the actuator can switch its output between the power levels $P_{1}$ and $P_{2}$, heating the mass $M$ which loses an intermediate amount of power $P_{\mathrm{E}}$ with the environment, so that a dynamic stationary regime is reached, with the thermometer measuring fluctuation between two extreme values $T_{\mathrm{L}}$ and $T_{\mathrm{H}}$

temperature $T(M)$, compared with two threshold values (high, $T_{\mathrm{H}}$, and low, $T_{\mathrm{L}}$ ), and the heater changes its power output between $P_{1}$, if $T(M)>T_{\mathrm{H}}$, and $P_{2}$, if $T(M)<T_{\mathrm{L}}$, where $P_{1}<P_{2}$ are the nearest digital approximations to $P_{E}$. The minus sign in Fig. 8 is due to the negative feedback used for stabilization: if the system temperature increases, the output power is decreased, and vice versa.

The condition for static thermal equilibrium requires that the power loss $P_{\mathrm{E}}$ is equal to one of the power output states of the heater, $P_{1}$ or $P_{2}$. In all other cases, an oscillating steady state is reached. When the current temperature is higher than the lower threshold $T_{\mathrm{L}}$, the input power is $P_{1}$, and the mass suffers a net power loss $P_{\mathrm{E} 1}=P_{\mathrm{E}}-P_{1}$; therefore, its temperature decreases at a rate depending upon its thermal capacity $C_{\mathrm{T}}$. After some time, the descending temperature reaches the threshold value $T_{\mathrm{L}}$, so that the heater switches to output power $P_{2}$, and the system, now heated by a constant flux of energy $P_{2 \mathrm{E}}=P_{2}-P_{\mathrm{E}}$, begins to warm up until reaching temperature $T_{\mathrm{H}}$. After that, the heater switches to $P_{1}$ again and the cycle is repeated. The variation between $T_{\mathrm{H}}$ and $T_{\mathrm{L}}$ is associated to an amount of energy $\Delta E=C_{\mathrm{T}} \Delta T$, where $\Delta T=T_{\mathrm{H}}-T_{\mathrm{L}}$, in both warmup and cool-down phase, i.e. $\Delta E=P_{\mathrm{E} 1} \tau_{\mathrm{f}}=P_{2 \mathrm{E}} \tau_{\mathrm{r}}$. This defines the rise and fall time, $\tau_{\mathrm{f}}=C_{\mathrm{T}} \Delta T / P_{\mathrm{E} 1}$ and $\tau_{\mathrm{r}}=C_{\mathrm{T}} \Delta \mathrm{T} / P_{2 \mathrm{E}}$, respectively, as well as the oscillation period: $\tau_{\mathrm{o}}=\tau_{\mathrm{f}}+\tau_{\mathrm{r}}$. The period becomes indefinitely long as one of the power output levels approaches $P_{\mathrm{E}}$, i.e. stationary thermal equilibrium. The average temperature of the system is $\langle T(M)\rangle=\left(T_{\mathrm{H}}+T_{\mathrm{L}}\right) / 2$, as $P_{1}$ and $P_{2}$ are the best digital approximations to the desired value $P_{\mathrm{E}}$.

The simple model can be applied to our CCD camera head. We assume a rigid detector, displaced from its nominal position due to the deformation of its metal supporting structure, having a thermal expansion coefficient about five times larger than silicon. The Peltier cell resolution is $\Delta T \simeq 0.1{ }^{\circ} \mathrm{C}$, and we assume for the cold node a thermal capacity of $\sim 10 \mathrm{~J} /{ }^{\circ} \mathrm{C}$ (equivalent to $0.02 \mathrm{~kg}$ of iron or copper, or $0.01 \mathrm{~kg}$ of aluminum). The energy involved in the thermal cycle is $\Delta E=C_{\mathrm{T}} \Delta T \simeq 1 \mathrm{~J}$; we 
assume $P_{\mathrm{E}}=\frac{1}{2}\left(P_{1}+P_{2}\right)$, so that the rise and fall time are equal. With a full-range cooling power of $2.5 \mathrm{~W}$, and $8 \mathrm{bit}$ resolution, the output step is $\Delta P=P_{2}-P_{1}=10 \mathrm{~mW}$. Then, the rise/fall time is $\tau_{\mathrm{f}}=\tau_{\mathrm{r}} \simeq 200 \mathrm{~s}$, corresponding to an oscillation period $\tau_{\mathrm{o}} \simeq 400 \mathrm{~s}$, or little more than 6 min, consistent with the observed time scale.

Moreover, the linear expansion coefficient is $\gamma \simeq 13$ parts per million per degree $\left(\mathrm{ppm} /{ }^{\circ} \mathrm{C}\right)$ for some steels and $\sim 17 \mathrm{ppm} /{ }^{\circ} \mathrm{C}$ for copper; therefore, the linear deformation of a metallic component in the cold head, with length $l=$ $0.05 \mathrm{~m}$, is $\Delta l=l \gamma \Delta T=6.5 \div 8.510^{-8} \mathrm{~m}$, quite consistent with the measured fluctuations in the raw data: $1 \%$ of the $9-\mu \mathrm{m}$ pixel is actually $90 \mathrm{~nm}$ ! Therefore, our simple model provides qualitative agreement with the systematic effects observed in the raw data, considering only the thermal behavior of the CCD head.

\section{Extrapolation to the GAIA detector}

The GAIA detection system will be endowed with a large mosaic composed of several hundred CCDs, each with a dedicated analog output line. The CCD output circuit is usually an on-chip MOS source follower, with a DC operating point defined by the bias network, and the charge packet from each CCD pixel appears as a modulation of the output voltage level. In static conditions, the power dissipation on the detector is stable; however, GAIA operates in continuous readout mode, because of the TDI observation. Since the star density and brightness are extremely variable over the sky, the signal level at the CCD output is not stable. The readout of a bright star signal corresponds to a perturbation of the power dissipation on the CCD, which usually consists in a net transfer of energy from the output amplifier to the external load.

This small internal dissipation is the only term to be taken into account for GAIA, as the design includes passive cooling for the CCD mosaic and provides a very stable thermal environment. For a single target, assuming a charge responsivity of $4 \mu \mathrm{V} / \mathrm{e}^{-}$and a peak signal of $10^{5}$ electrons, the output voltage swing is $0.4 \mathrm{~V}$. With a DC bias current of $0.5 \mathrm{~mA}$, and in the simplified assumption of a sinusoidal signal, the modulated power is $W=\frac{1}{2} V_{\mathrm{s}} I_{\mathrm{L}} \simeq 0.1 \mathrm{~mW}$. As the readout time for a single star is $\sim 50 \mu$ s (five pixels), the energy contributed is $510^{-9} \mathrm{~J}$, to be compared with the value of $\sim 1 \mathrm{~J}$ for the simple laboratory model described in the previous section. Even for the limiting case of a sky region with "infinite" object density, the energy perturbation contributed by each CCD on GAIA will be 100 times smaller than those experienced in our laboratory: $0.1 \mathrm{~mW}$ vs. $10 \mathrm{~mW}$.

The focal plane perturbation is therefore reduced from $\sim 1 \%$ to the order of $10^{-4}$ of the $9 \mu \mathrm{m}$ pixels, i.e. to about $1 \mathrm{~nm}$, even assuming the same structure stability as for our commercial CCD camera. With a plate scale of 4 arc$\mathrm{sec} / \mathrm{mm}$ (as currently planned), this perturbation is then $4 \mu$ arcsec, i.e. below the final mission accuracy specification of $10 \mu$ arcsec. Moreover, an infinite star density is not a realistic case, and the focal plane structure can be designed with much better dimensional stability than our commercial CCD camera, so that the geometry sensitivity to the thermal variation may be further depressed. Therefore, thermal effects onto the detector probably can be excluded as limiting factors for the final mission accuracy of GAIA.

\section{Conclusions}

Within the limits of our experiment, the operating principle of GAIA is not refuted: the measurement of relative positions on individual images from a CCD focal plane appears compatible with the precision level desired for bright stars, even without the advantage of TDI operation. The limiting accuracy in the determination of target positions in CCD images does not appear to be limited by the finite pixel size, at least down to the level of $1 / 1000$ of its value, and the underlying mathematical framework seems to be adequate at this level. The one-dimensional coordinate can then be translated into global astrometric positions through the observations of either a double instrument with relative orientation strictly monitored, or a single telescope fed by a beam combiner, repeatedly covering the whole sky for a few years, as in the Hipparcos scheme. At least in our set-up, which limits by design most of the known CCD limitations, a relative centering precision comparable with the requirements of GAIA, i.e. a dispersion of $1 / 700$ pixel for individual frames or $1 / 1300$ pixel for co-added images.

The paramount importance of an intrinsically stable design, within a quiet environment, is confirmed; in particular, temperature excursions of the order of $1 \mathrm{mK}$ over the focal plane would provide system errors comparable with the desired mission accuracy of GAIA, if the structure sensitivity is comparable to that of our experiment. The effects evidenced in our data are common mode image displacements (about 1/100 pixel), which in real cases may be related to perturbations of the satellite attitude or of the instrument optics. Correction for the common mode motion provide suppression of the disturbances by up to an order of magnitude. Therefore, simple calibration procedures promise to be effective.

Acknowledgements. The experiment concept and rationale benefitted from discussions with members of the Scientific Advisory Group appointed by ESA in support to refinement of the GAIA mission concept. C.O.M.P. (Cusano Milanino, MI, Italy) helped in development of the pinhole mask and supplying engineering information on some optical components. The engineers of Alenia Spazio contributed to the set-up implementation. Funding of our experiment was granted by the Italian Space Agency (ASI, contracts ASI ARS 96-77 and ASI ARS 98-92) for preliminary study of the GAIA mission. We also acknowledge a contribution from the National Council for Astronomy and Astrophysics (CNAA, contract CNAA 16/97) for part of the laboratory equipment. The layout of this paper benefits of the corrections and suggestions of our Referee.

We wish to express our thanks to all of them, as well as to the staff of the Observatory and the other institutes involved. 


\section{References}

Anderson, J., \& King, I. R. 1999, PASP, 111(763), 1095

Gai, M., Guarnieri, M. D., \& Lattanzi, M. G. 1997, Exp. Astr., $7(2), 87$

Gai, M., Casertano, S., Carollo, D., \& Lattanzi, M. G. 1998, PASP, 110(794), 848

Gai, M., Carollo, D., Delbò, M., Lattanzi, M. G., \& Massone, G. 1999, Proceedings of the 4th ESO Workshop on Optical Detectors for Astronomy, 13-16 September 1999, in press

Gilmore, G. F., Perryman, M. A. C., Lindegren, L., et al. 1998, Proceedings of the SPIE, vol. 3350, Astronomical Interferometry, 541

Holtzman, J. A., Hester, J. J., Casertano, S., et al. 1995, PASP, 107(708), 156

Horner, S. D., Germain, M. E., Greene, T. P., et al. 1998, AAS, Meeting 193, 12.06

Lattanzi, M. G., Gai, M., Cecconi, M., Cesare, S., \& Mana, G. 1997, Proceedings of the 3rd international Conference on Space Optics, Toulouse, 2-4 December, 1997
Lindegren, L. 1978, Proceedings of the IAU Colloquium No. 48, ed. F. V. Prochazka, \& R. H. Tucker, Modern Astrometry, 197

Lindegren, L., \& Perryman, M. A. C. 1996, A\&A, 116, 579

Loiseau, S., \& Shaklan, S. 1995, A\&A, 117, 167

Perryman, M. A. C. 1997, Proceedings of the HIPPARCOS Venice '97 Symposium, I, 13-16 May, 1997, ESA SP-402

Saint-Pé, O., Proceedings of the 4th ESO Workshop on Optical Detectors for Astronomy, 13-16 September, 1999, in press

Shao, M. 1998, Proceedings of the SPIE, vol. 3350, Astronomical Interferometry, 536

Smart, R. L., Bucciarelli, B., Lattanzi, M. G., Massone, G., \& Chiumiento, G., A\&A, 348, 653

van Leeuwen, F., \& Perryman, M. A. C. 1995, Proceedings of Future Possibilities for Astronomy in Space (A Joint RGOESA Workshop), Cambridge, UK, 19-21 June, 1995, ESA SP-379

Whitmore, B., Heyer, I., \& Casertano, S. 1999, PASP, 111(766), 1559 